\title{
High-speed Digitization Readout of Silicon Photomultipliers for Time of Flight Positron Emission Tomography
}

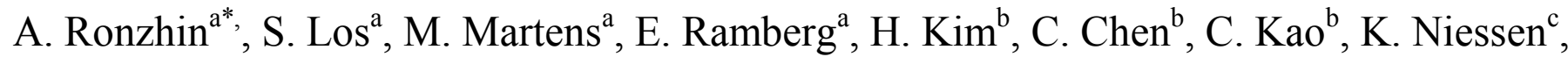 \\ A. Zatserklyaniy ${ }^{\mathrm{d}}$, M. Mazzillo ${ }^{\mathrm{e}}$, B. Carbone ${ }^{\mathrm{e}}$, G. Condorelli $^{\mathrm{e}}$, P. Fallica ${ }^{\mathrm{e}}$, A. Piana $^{\mathrm{e}}$, \\ D. Sanfilippo ${ }^{\mathrm{e}}$, G. Valvo ${ }^{\mathrm{e}}$ \\ ${ }^{a}$ Fermi National Accelerator Laboratory, Batavia, IL 60510, USA, \\ ${ }^{b}$ University of Chicago, Chicago, IL 60637, USA, \\ ${ }^{c} S U N Y$ of Buffalo, NY 14260, USA, \\ ${ }^{\mathrm{d}}$ University of Puerto Rico, Mayaguez, Puerto Rico 00681 USA, \\ ${ }^{\mathrm{e}}$ STMicroelectronics, Catania, 95121, Italy
}

Abstract. We report on work to develop a system with about 100 picoseconds (ps) time resolution for time of flight positron emission tomography [TOF-PET]. The chosen photo detectors for the study were Silicon Photomultipliers (SiPM's). This study was based on extensive experience in studying timing properties of SiPM's [1-4]. The readout of these devices used the commercial high speed digitizer DRS4 [5]. We applied different algorithms to get the best time resolution of 155 ps Guassian (sigma) for a LYSO crystal coupled to a SiPM. We consider the work as a first step in building a prototype TOF-PET module.

\section{Introduction}

The field of positron-emission-tomography (PET) has been rapidly developing. But there are significant limitations in how well current PET scanners can reconstruct images, related to how fast data can be acquired, how much volume they can image, and the spatial and temporal resolution of the generated photons. Typical modern scanners now include multiple rings of detectors, which can image

Manuscript received February 17, 2011. Anatoly Ronzhin is with Ferm National Accelerator Laboratory, Batavia, IL 60510 USA (telephone: 630840-8630, e-mail: ronzhin@fnal.gov). a large volume of the patient. In this type of scanner, one can treat each ring as a separate detector and require coincidences only within the ring, or treat the entire region viewed by the scanner as a single 3 dimensional volume. This $3 \mathrm{~d}$ technique has significantly better sensitivity since more photon pair trajectories are accepted. However, the scattering of photons within the volume of the patient, and the effect of random coincidences limits the technique. The advent of sub-nanosecond timing resolution detectors means that there is potentially much better rejection of scattered photon events and random coincidence events in the 3D technique. In addition, if the timing is good enough, then the origin of photons pairs can be determined better, resulting in improved spatial resolution - so called 'Time-of-Flight' PET, or TOF-PET. Currently a lot of activity has occurred in applications of SiPMs for TOF-PET. [6-8]. This is due to the devices' very good time resolution, low profile, lack of high voltage needed, and their non-sensitivity to magnetic fields. While investigations into this technique have begun elsewhere, we feel that the extensive SiPM characterization and data acquisition expertise of Fermilab, and the historical in-depth research of PET imaging at University of Chicago will combine to make significant strides in this field. We also benefit by a working relationship with the SiPM producer STMicroelectronics (STM) $[3,4]$. 


\section{SiPM Layout, role of clipping capacitance}

We present here results obtained from SiPM's produced by STM, as well as devices obtained from Hamamatsu. The shape of the SiPM signal plays an important role for improvement of the time resolution [9]. During an extensive series of timing tests we have performed on SiPM's, we would often use a capacitive coupling $(10 \mathrm{pF})$ to clip the slow tail of the SiPM response (see Figure 1 for a schematic). This was performed due to the input requirements of the Ortec 9327 constant fraction discriminator unit we use. Figure 2 presents the effect of the capacitance as an illustration. The use of a high bandwidth amplifier if needed, was shown not to change the pulse shape. The STM SiPM was illuminated by $405 \mathrm{~nm}$ light coming from a high speed PiLas laser. For this light input, the signal was suppressed by a factor of 30 due to the capacitive coupling. For the TOF-PET application the shape of the signal is a significant issue. The sharpening of the signal (including its rise time) due to the coupling should improve the time resolution, but it can worsen the energy resolution. That is why we investigated the influence of the clipping capacitance and preamplifiers on the time and energy resolution. The Pomona boxes with SiPMs, circuit boards and mechanics for radiator (or crystal) support is shown in fig. 3 .

\section{Pulser and Laser Data}

The schematic diagram of the readout with PiLas laser as light source is shown in fig. 4 . The DRS4 is a $5 \mathrm{GHz}$ digitizer developed by Stefan Ritt at Paul Scherrer Institute, Switzerland [4], and was used to in the measurements reported here. We used the version of the DRS4 with 4 input channels. The DRS4 allows a digitization of the input with a sampling rate of $5 \mathrm{GS} / \mathrm{s}$ with an individual channel depth of 1024. The schematic diagram of the readout with the DRS4 is shown in fig. 8 . Sometimes we used Ortec V120C to amplify the SiPM signals to fit the DRS4 dynamic range.

First we measured the DRS4 electrical time resolution. The PiLas NIM output was used as trigger for the DRS4. Another NIM signal was split with a high bandwidth splitter into two identical signals. Each half was applied to channels 1 and 2 of the DRS4. The linear fit on the leading edge of the signals was applied (fig. 5). The fit value for the time at the signals half maximum was taken and the time difference between these 2 values was analyzed. This very simple algorithm was used as a starting point for our analysis. The time spectrum we obtained demonstrated a Gaussian distribution with 8 picoseconds sigma.

The same procedure was repeated with STM signals instead of NIM pulses. The two STM SiPMs were placed at the same distance from the PiLas laser head and were illuminated by the laser light. The STMs overvoltage was 5 Volts and both were operated under room temperature which was $+25^{\circ} \mathrm{C}$. The PiLas light signal was 33 ps full width at half maximum, the wavelength was $405 \mathrm{~nm}$, most data taken with the repetition rate of $100 \mathrm{~Hz}$. We could change the light intensity by the "TUNE" laser option, also as by applying optical filters between the laser head and the SiPMs. The data were taken with the $10 \mathrm{pF}$ capacitance in place. The pulse height distribution $(\mathrm{PH})$ was obtained by integrating the charge collected over all time bins in each DRS4 sample. A few tens of samples before the signal appearance were taken to estimate the base line of the signal. The measured number of photoelectrons (PE) was about 25 (Figure 6). The number of PE was obtained by measuring the $\mathrm{PH}$ distribution and fitting it with a Gaussian. The width of the Gaussian fit will indicate the statistical nature of the PE spread. For this laser data, the time resolution with $100 \mathrm{PE}$ was $18 \mathrm{ps}$ and with $25 \mathrm{PE}$ was $39 \mathrm{ps}$, which roughly follows the expected inverse square root dependence of the timing on the number of PE. This very simple initial approach revealed that the result we obtained for the laser data is consistent with the earlier NIM pulse results [1]. The time resolution without the $10 \mathrm{pF}$ capacitance, using the leading edge approximation, was about the same. These preliminary results showed that best time resolution corresponds to when there is $60-400$ $\mathrm{mV}$ maximum signal amplitude, corresponding to the mid-range of the DRS4 digitizer. We also found that the time resolution for this data is dependent on the algorithm we used. The issue will be considered in more detail below. 
IV. PET Setup, with Radioactive Source Data

Figure 7 shows the external view of the experimental TOF-PET setup. It consists of two SiPMs with optically attached crystals inside of Pomona boxes. These devices face a Na-22 radioactive source from opposite sides. A Keithley 2410 power supply used to bias the SiPMs [10]. As with the previous laser and NIM pulse data, the signals from the SiPMs were split into two halves, with one half going to form a coincidence trigger and the other half being digitized by the DRS4. We measured time and pulse height distributions with varying:

1. SiPMs: We used STM and Hamamatsu devices. The STM sensitive area is $3.5 \times 3.5$ $\mathrm{mm}^{2}$, with a pixel count of 3,600 , pixel size $58 \times 58 \mathrm{um}^{2}$, photon detection efficiency (PDE) about 18\% for blue light, a breakdown voltage $\sim 28$ Volts, gain of up to $10^{6}$, overvoltage of 5 Volts. The Hamamatsu sensitive area is $3 \times 3 \mathrm{~mm}^{2}$, pixel count 3,600 , pixel size $50 \times 50 \mathrm{um}^{2}, \mathrm{PDE}$ is about $40 \%$ for blue light, breakdown voltage $\sim 70$ Volts, gain is up to $10^{6}$, overvoltage is up to 2.5 Volts.

2. Crystals consisting of LYSO of different size. The LYSO crystals sizes were, $3 \times 3 \times 10 \mathrm{~mm}^{3}$, $3 \times 3 \times 15 \mathrm{~mm}^{3} 3 \times 3 \times 20 \mathrm{~mm}^{3}$ and $2 \times 2 \times 7 \mathrm{~mm}^{3}$.

3. Clipping capacitance and without it.

4. Radioactive source Co60.

5. Radioactive source $\mathrm{Na} 22$.

We found the shape of the signals does not depend on the crystal size we used. The SiPMs coincidence counting rate was about $30 \mathrm{~Hz}$ for both Co60 and $\mathrm{Na} 22$ sources. The distance between the 2 counters was minimal to place the sources between them and was slightly increased to accommodate the larger crystals size. We found the larger distance does not change significantly time and pulse height resolution with only going down the coincidence counting rate. Optical grease was used to get a good optical contact between the crystals and SiPMs. The rest of the LYSO sides were covered by a few layers of thin TEFLON tape.

The shape of the signals was strongly dependent on whether we used clipping capacitance or not. The traces of STM and Hamamatsu signals with 10 $\mathrm{pF}$ clipping capacitance and without it are shown in fig. 9.

The examples of the time and $\mathrm{PH}$ distributions are presented in fig. 10. The best time resolution obtained was 155 ps with events corresponding to the 1.17- $1.33 \mathrm{MeV}$ energy of the Co60 photon energy. A cut in $\mathrm{PH}$ distribution was applied to retain only the photoelectric interaction peak.

The conditions were: Hamamatsu SiPM, 2x2 $\times 7$ $\mathrm{mm}^{3}$ LYSO crystal, VT120C amplifier, Co60 radioactive source.

We performed the same test with $\mathrm{Na} 22$ source. The best time resolution obtained was 244 ps for MPPCs and 334 ps for STM with LYSO crystals, $3 \times 3 \times 15 \mathrm{~mm}^{3}$ crystals in both cases. The data are taken with $10 \mathrm{pF}$ of the clipping capacitance. The examples of the time and $\mathrm{PH}$ distributions are presented for MPPC in fig. 11, and for STM in fig. 12. MPPC and STM results obtained with the same pulse function approach (see below).

\section{Algorithms}

We started the data analysis with fitting to the leading edge of the signals by straight line, as we discussed above. The point at half maximum of the signal amplitude was detected and used as the timing point. The spectra of the time difference for the two SiPMs signals by using these points were analyzed first. Another analysis was performed such that we fit where the straight line from the fit crossed the fit signal base line. This approach was applied both to data obtained with the laser and with a radioactive source. As a result of these investigations we found that the natural shape of the signals leading edge is far from a straight line, especially for data with the radioactive source and without any shaping of the signals. Even for short signals we observed different slopes along the leading edge. 
This is why a functional form approach was introduced. The function well describes different conditions, e.g. SiPM signals with and without the clipping capacitance. A simple model of a SiPM as charging/discharging capacitance was taken as a first approximation to the pulse function. The analytical expression for this function is:

$\mathrm{p}(\mathrm{t})=(1-\exp (-\mathrm{t} / \tau 1) * \exp (-\mathrm{t} / \tau 2)$,

where $\tau 1, \tau 2$ are charging and discharging time constants. To take into account experimental conditions (jitter, etc.) we convolute the pulse function with resolution function. We use gaussian resolution function with parameter $\sigma$ which represents an experimental resolution. We got significant improvement of the calibration time resolution ( 4 ps instead of $8 \mathrm{ps}$ ) by applying the function to the PiLas data. To analyze data obtained with LYSO crystal we convoluted the pulse function with exponent with parameter $\mathrm{T}$ which represents crystal decay time. Fit to crystal data gives a realistic value for $\mathrm{T}$ of about $40 \mathrm{~ns}$. We got a good fit of the function to the real SiPM signals for both PiLas and radioactive source data. The data from the LYSO crystal show significant fluctuations of the signal shape. We apply two stage approach to the crystal data. We fit pulse function to the whole signal to find position of the leading edge. Then we fix discharge time parameter $\tau_{2}$ and fit the leading edge region only.

\section{Discussion}

Both single photoelectron SiPm signal and crystal light pulses have very sharp leading edge and much slower falling tail. It was a bit surprising for us to see that convolution of the SiPms signal shape with crystal light pulse shape changes dramatically the leading edge of the resulting signal. Our result is turned out to be due to the long falling tail of the SiPms signals in accordance with [5]. Shortening of the SiPM signal with clipping capacitance allows get sharp leading edge. As consequence the time resolution improved. The "payoff" for that is the introduction of amplifiers into the system. Among tested algorithms the best time resolution obtained with the signal fitting by "pulse function". And the best timing point chosen was close to the linear part of the signal appearance. The time resolution obtained with $\mathrm{Na} 22$ radioactive source corresponds to the linear approximation of the leading edge at the level of $10-20 \%$ of the signal amplitude. We continue to study different algorithms for data analysis and results will be reported later on.

\section{Conclusion}

We performed an initial study of silicon photomultipliers as photodetectors with LYSO crystals for TOF-PET application. Our results were obtained with the DRS4 waveform digitizer and are consistent with previous results based on a constant fraction discrimination circuit (containing Ortec 9327, TAC567 and ADC114 modules). A few algorithms were investigated for time resolution analysis. The best time resolution obtained was with pulse function approximation of the signals. The time resolution improved with clipping of the SiPMs signals. We believe the obtained results can be improved. We consider this study as an initial step for design and production of our first TOF-PET module prototype [11].

Figures

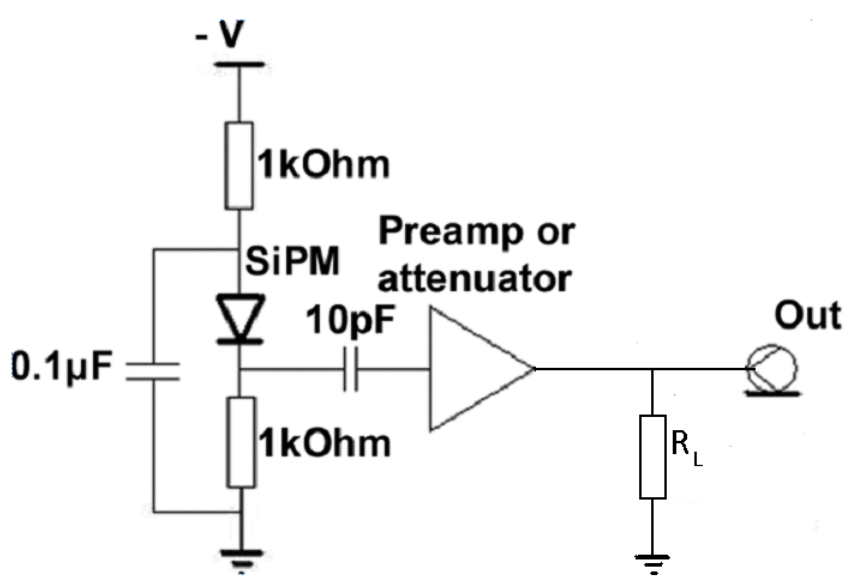


Fig. 1. Schematic of the biasing and readout circuit used for the SiPM timing measurements.
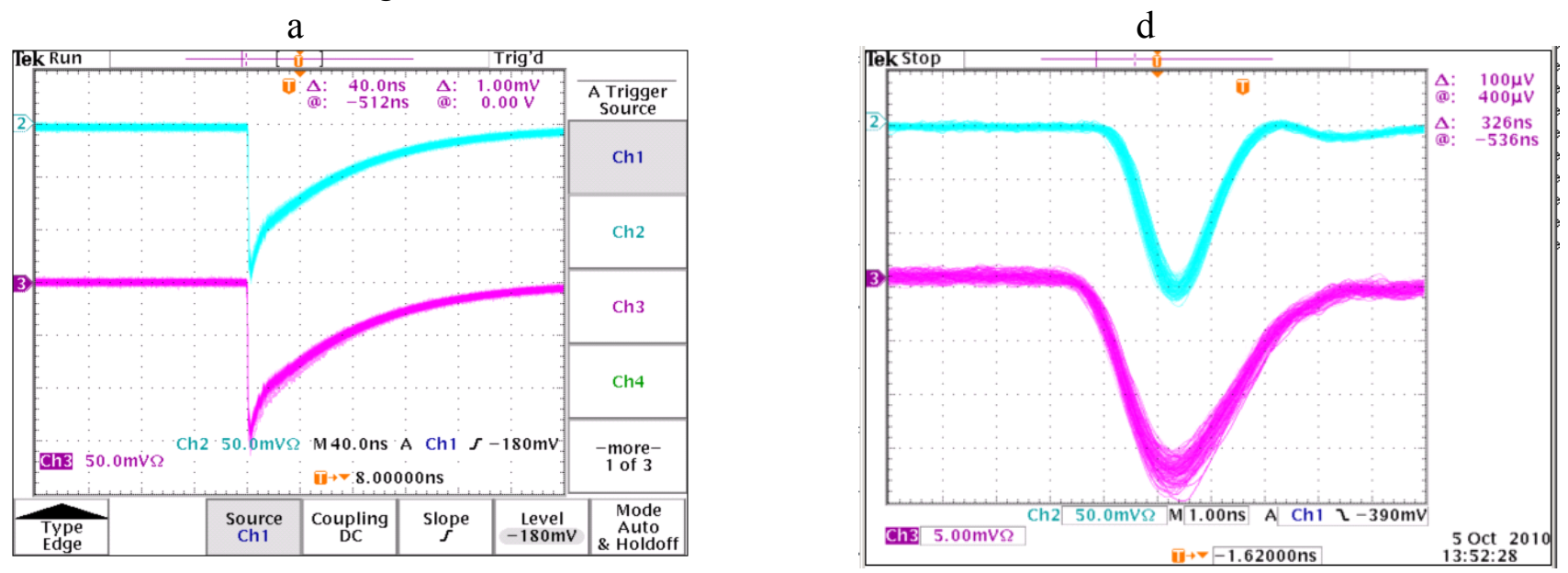

b
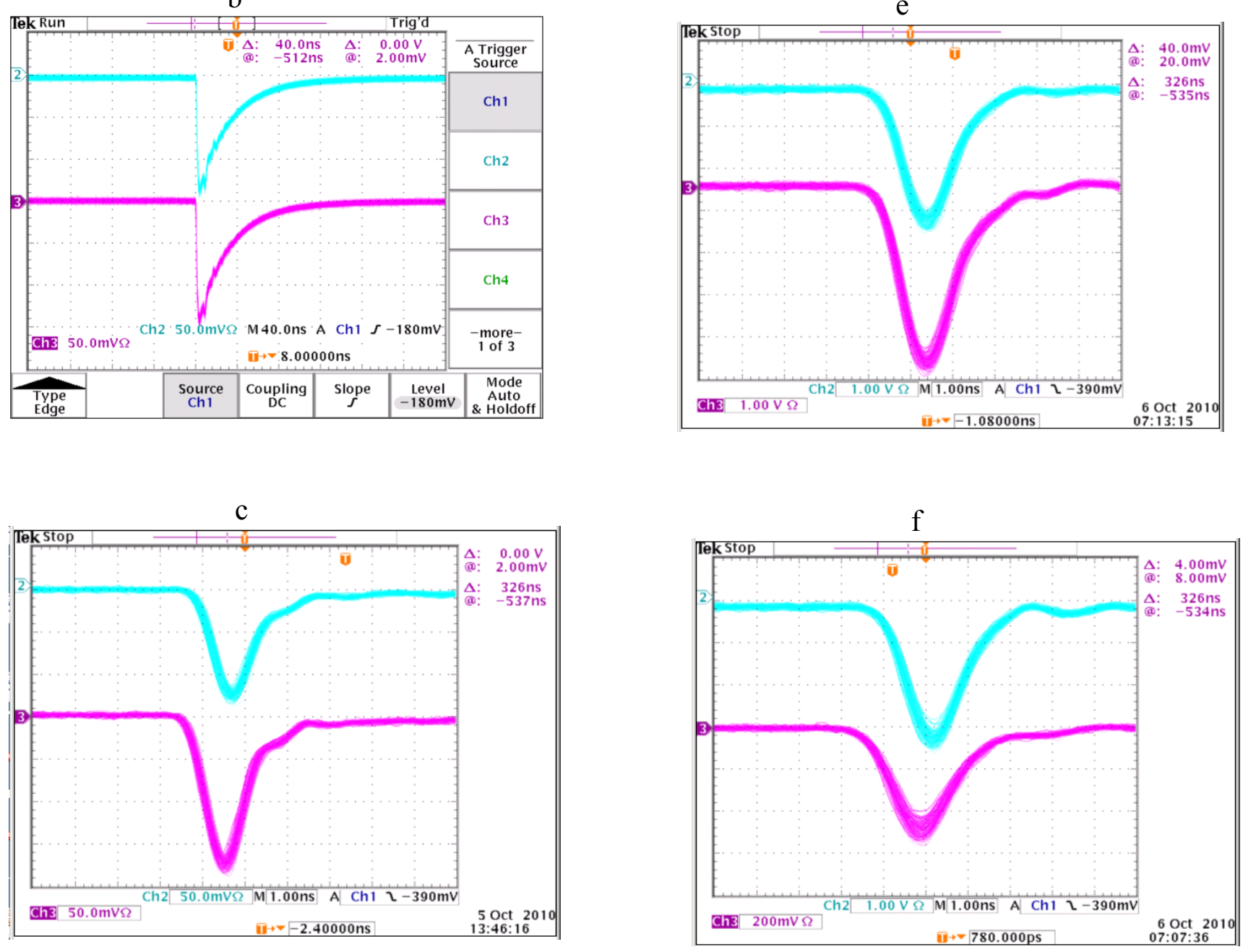

Fig. 2. Traces of the STM and MPPC signals. The SiPMs illuminated by PiLas laser head. Traces are taken without clipping capacitance $(a, b)$ and with it 
(c, d) and with Ortec VT120C preamplifier (e, f, 10 $\mathrm{pF}$ capacitance).

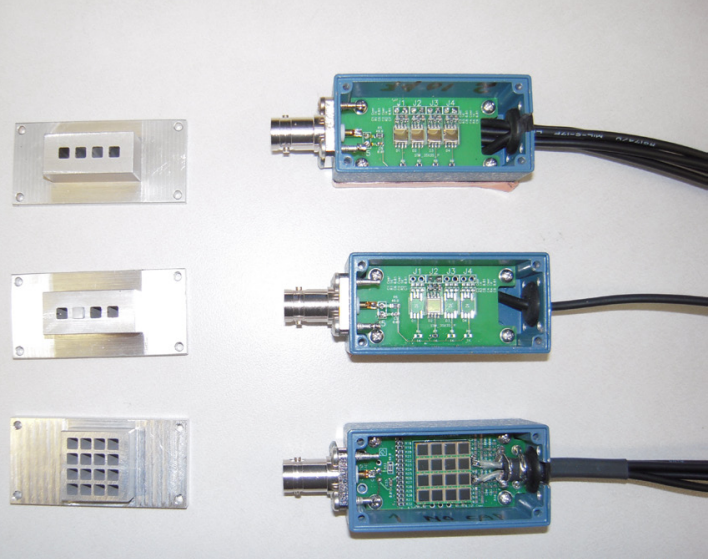

16. $12.2010 \quad 01: 13$

Fig. 3. From the top, on right: 4 STM inside the Pomona box with printed circuit board. The support for radiators or crystals is on the left. The same for 1 STM is in the middle. The MPPC matrix, $4 \times 4$ cells inside Pomona box with printed circuit board and support for radiators or crystals are on the bottom.

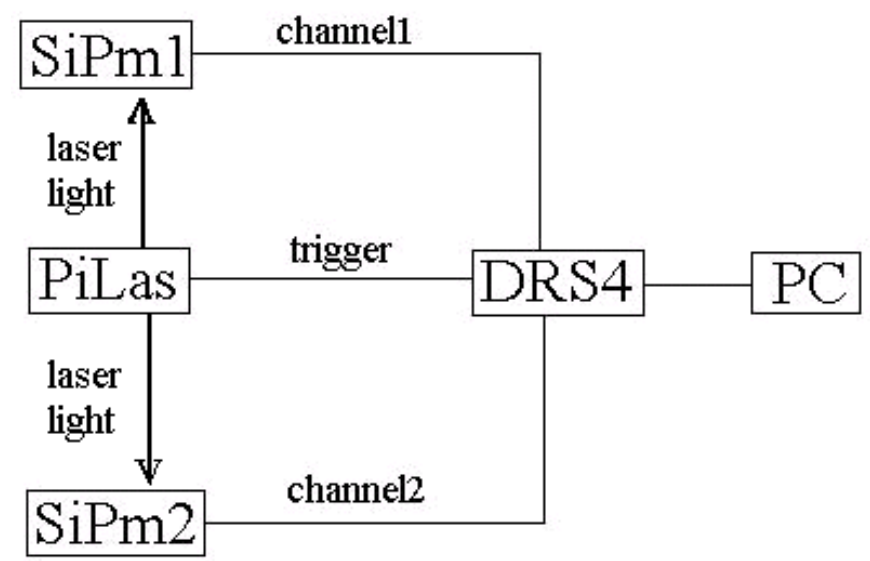

Fig. 4. The schematic diagram of the readout with the PiLas laser. SiPm1, 2 - silicon photomultipliers, PiLas - picoseconds laser, DRS4 - waveform digitizer, $\mathrm{PC}-$ personal computer. a

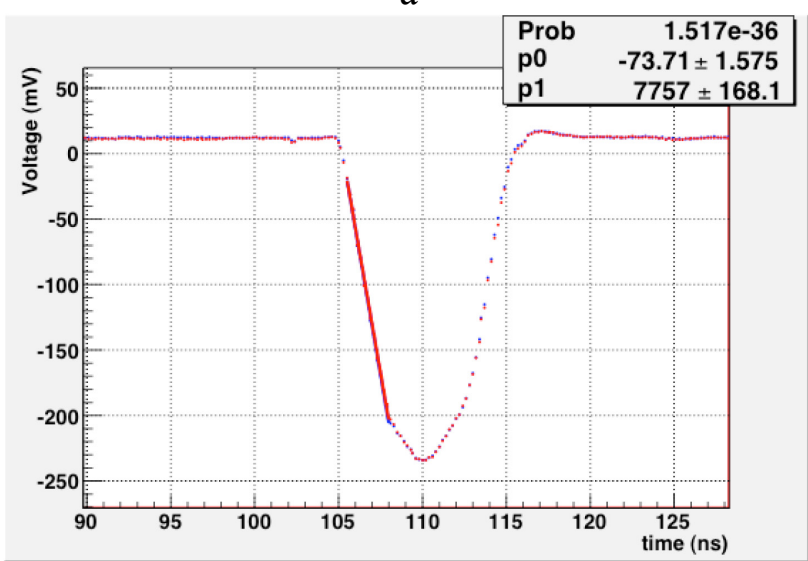

b

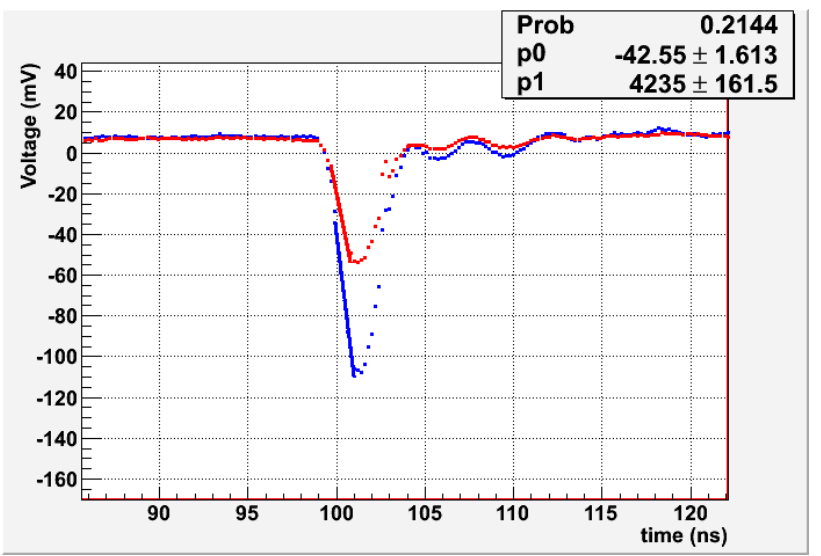

Fig. 5. Illustration for linear fit to the leading edge of the signal. $\mathrm{a}$ - attenuated NIM signal, $\mathrm{b}$ - signals of 2 STMs, illuminated by PiLas laser.

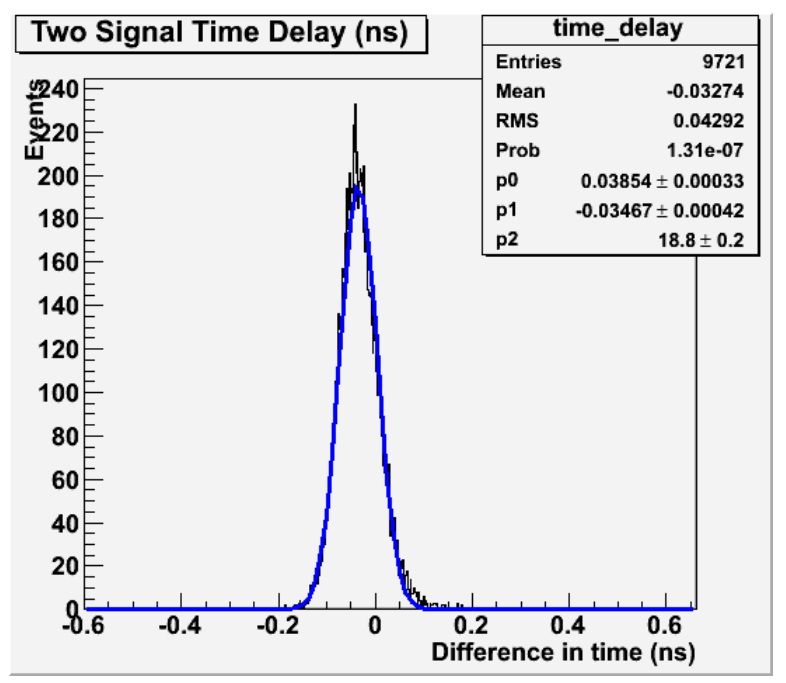


Fig. 6. Timing spectra, corresponding 25

photoelectrons. Time resolution (sigma) is $38.5 \mathrm{ps}$.
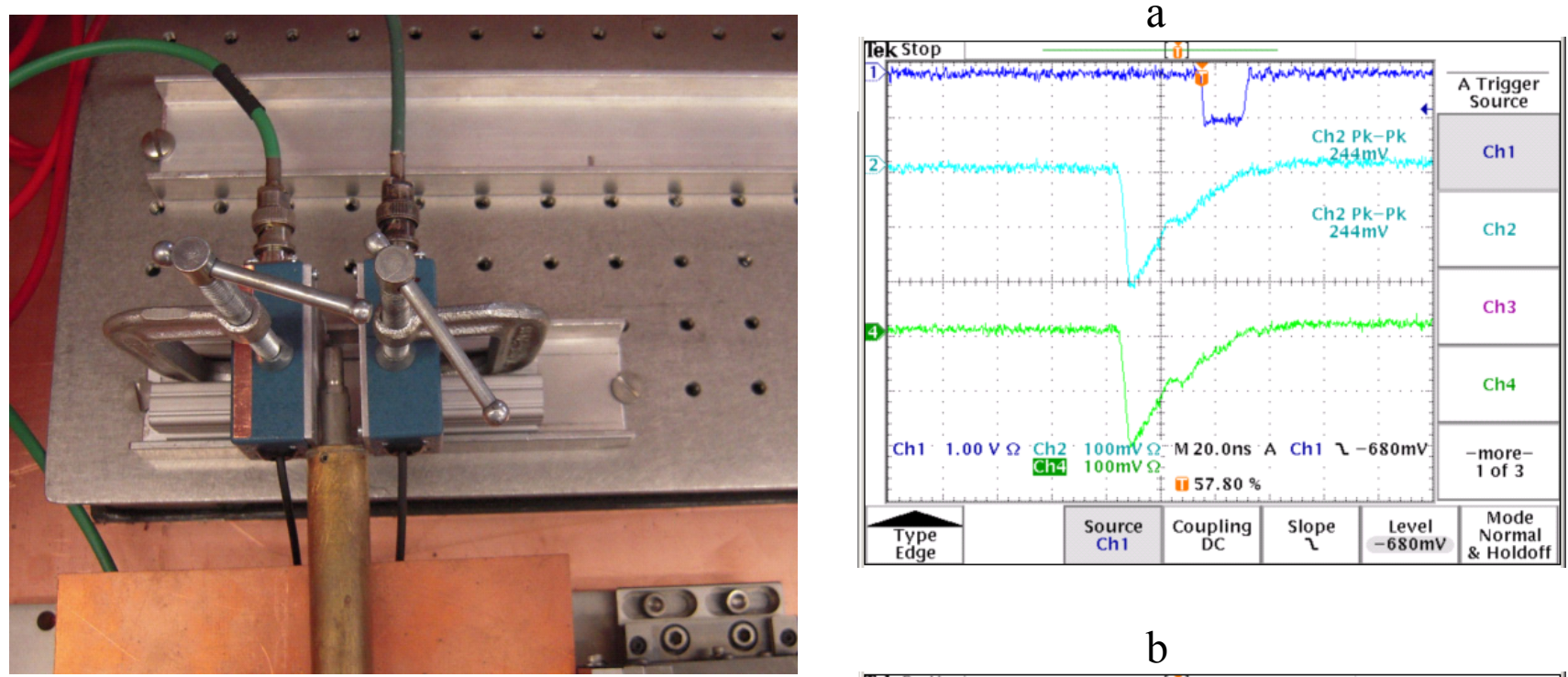

Fig. 7. External view of the TOF-PET setup.

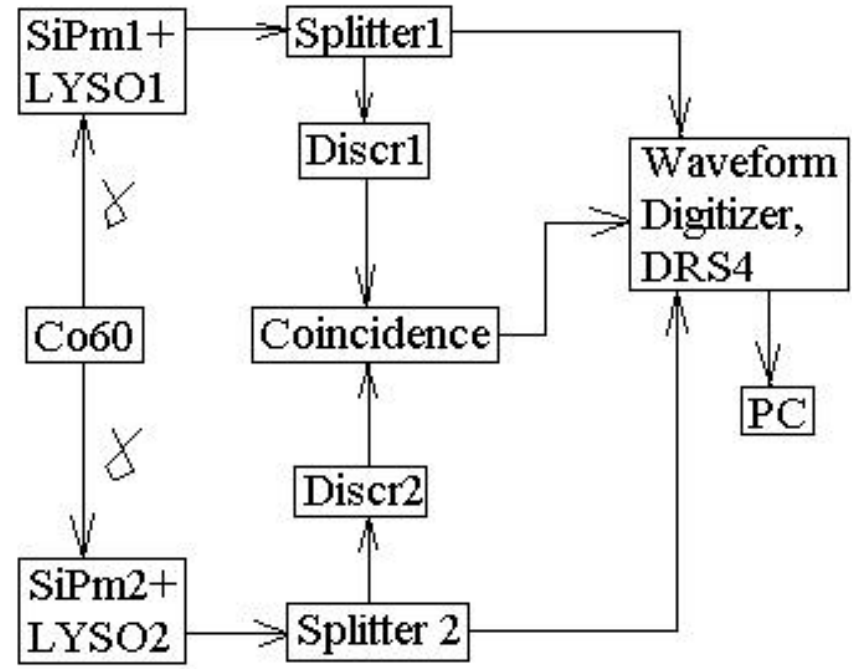

Fig. 8. The schematic diagram of the readout with the DRS4.
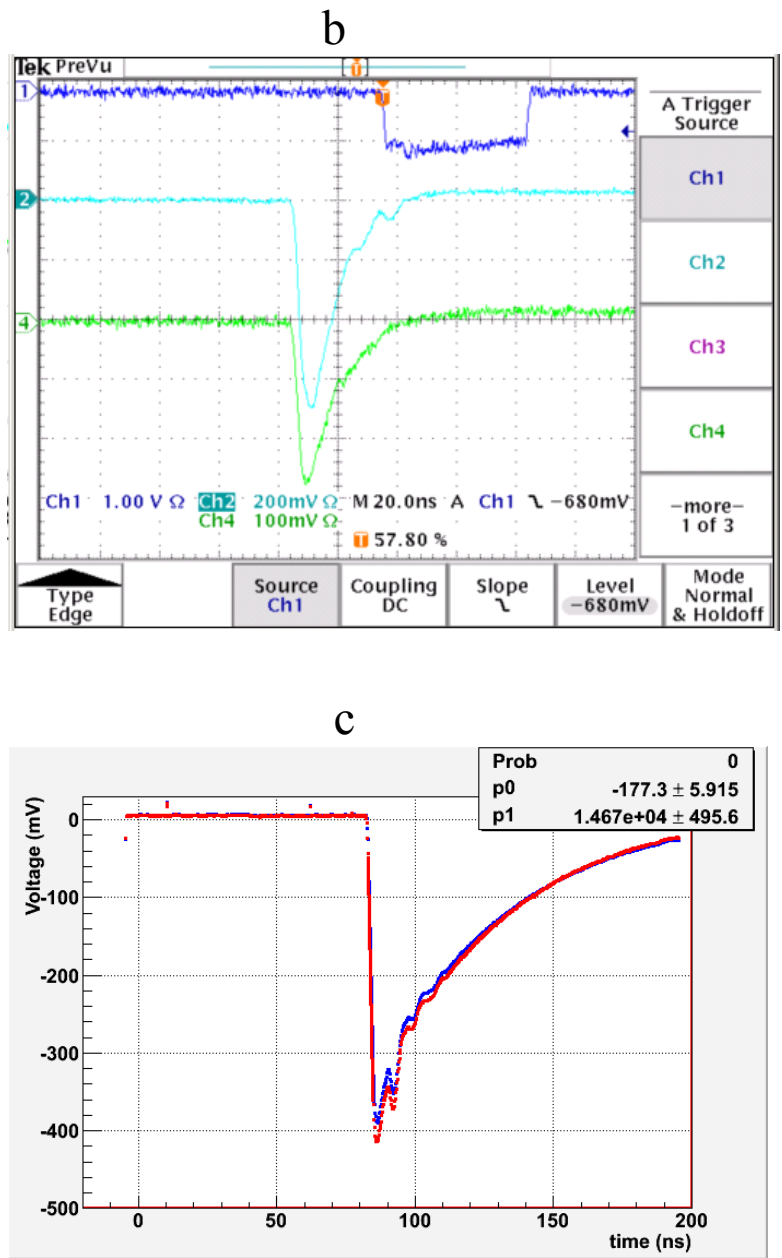

Fig. 9. Traces of 2 STMs signals (a), and 2 MPPC signals (b) taken by Tektronix oscilloscope. $10 \mathrm{pF}$ clipping capacitance. LYSO crystals irradiated by radioactive source Co60, Ortec VT120C preamplifiers; $\mathrm{c}$ - traces of 2 MPPCs signals taken 
with DRS4. No clipping capacitance. No Ortec VT120C preamplifier, Co60 source.
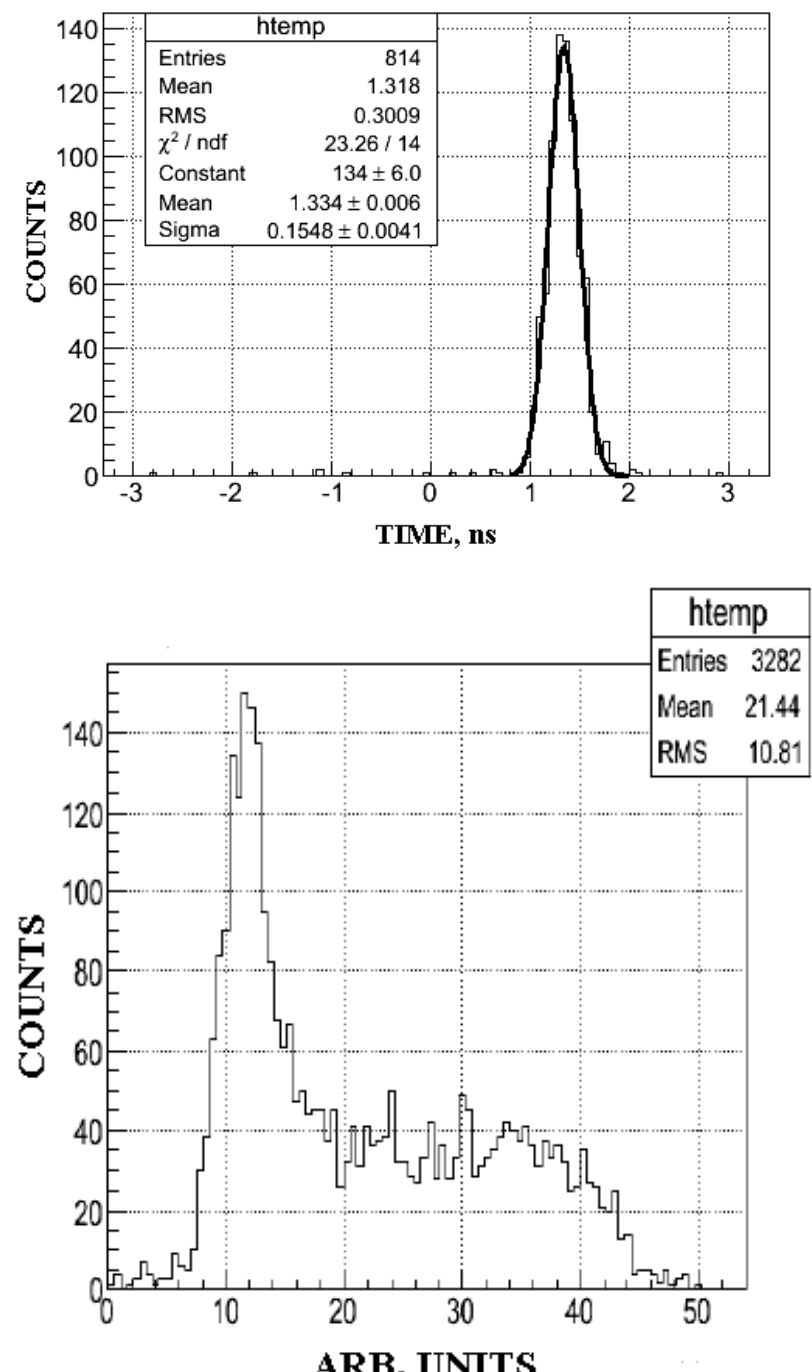

Fig. 10. Example of time (top) and pulse height (bottom) distribution. The conditions: Hamamatsu MPPC, $3 \times 3 \mathrm{~mm}^{2}$; LYSO crystal, $2 \times 2 \times 7 \mathrm{~mm}^{3}$; VT120C amplifier, Co60 radioactive source. 155 ps (sigma) time resolution.

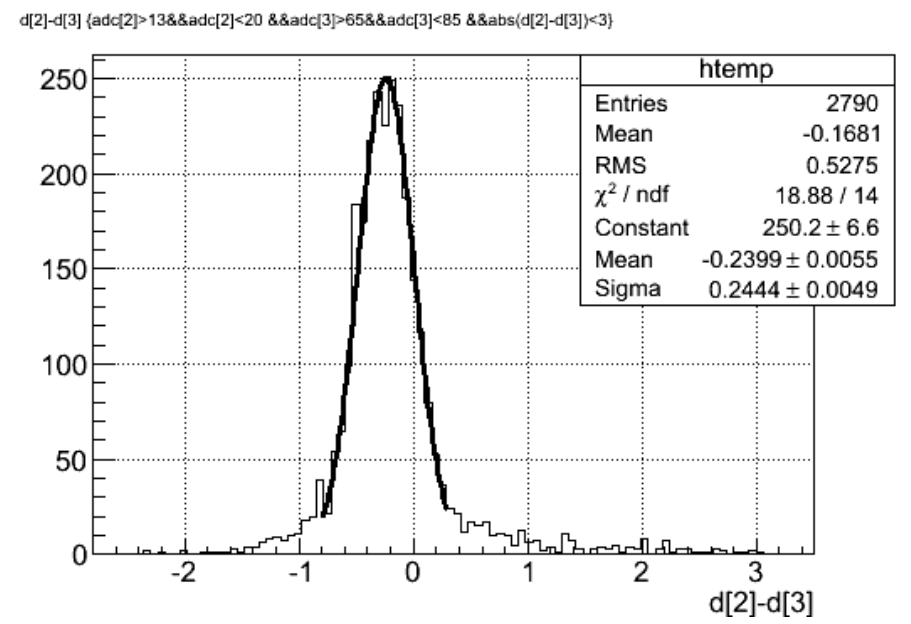

$\operatorname{adc}[3]\{\operatorname{adc}[3]>0 \& \& \operatorname{adc}[3]<1000\}$

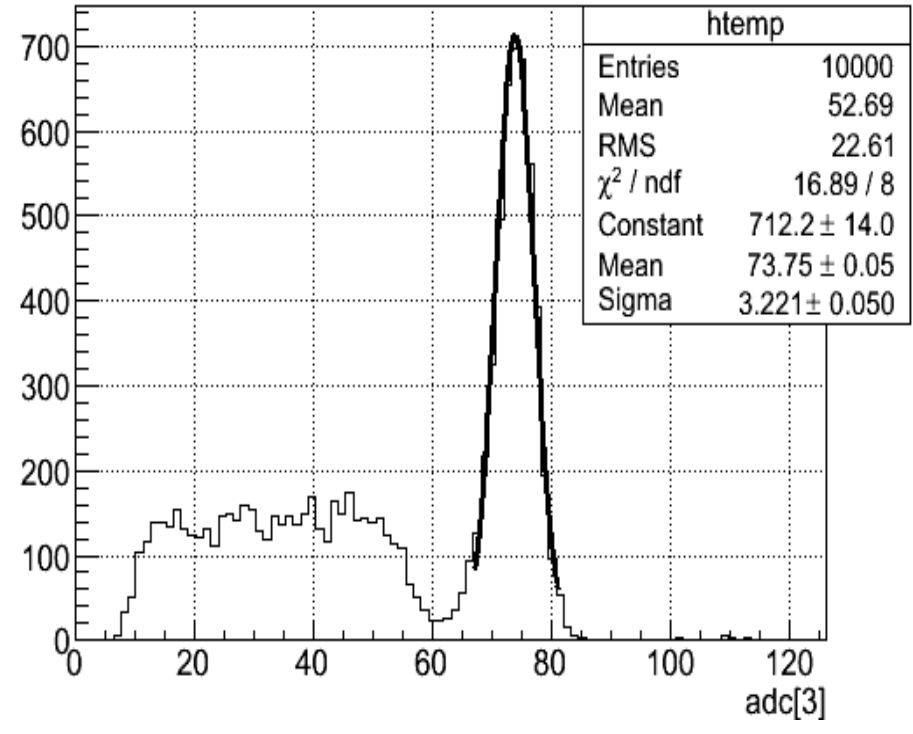

Fig. 11. Example of time (top) and pulse height (bottom) distribution. The conditions: Hamamatsu MPPC, $3 \times 3 \mathrm{~mm}^{2}$; LYSO cristal, $3 \times 3 \times 15 \mathrm{~mm}^{3}$; VT120C amplifier, Na22 radioactive source. 244 ps (sigma) time resolution, 10\% (FWHM) photo peak resolution. 


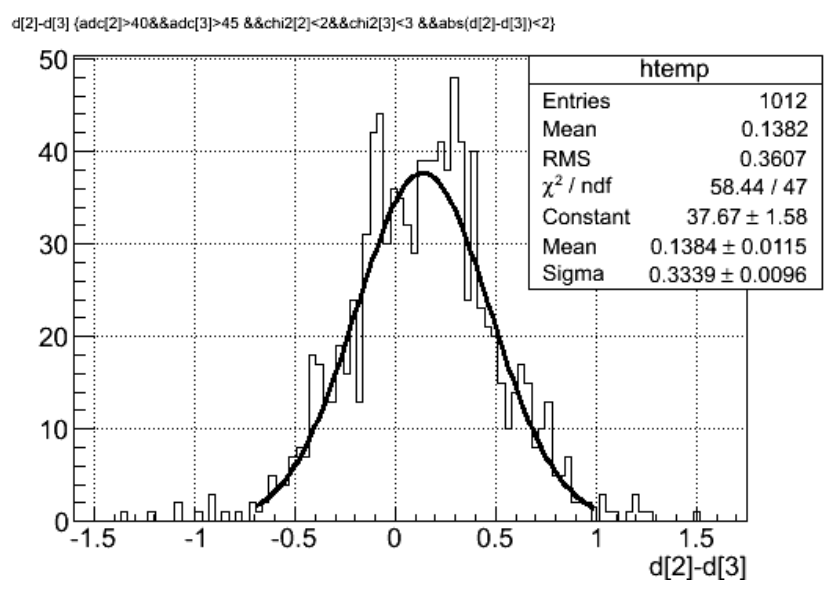

$\operatorname{adc}[2]\{\operatorname{adc}[2]>0 \& \& \operatorname{adc}[2]<70\}$

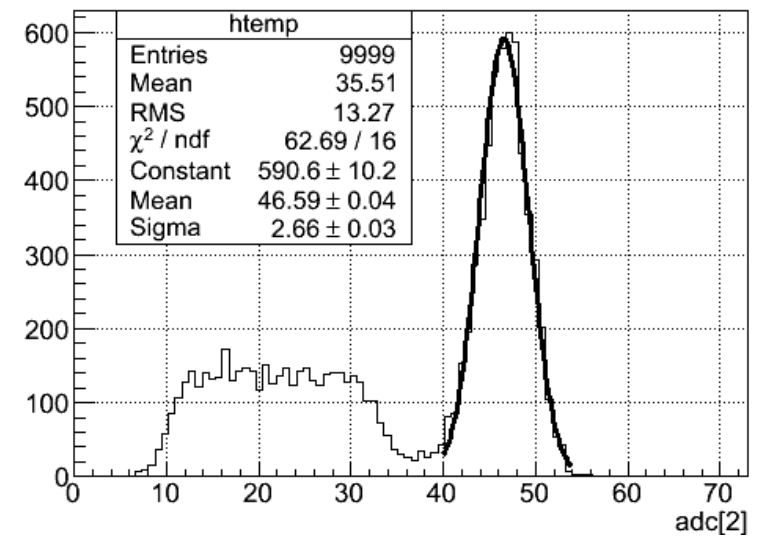

Fig. 12. Example of time (left) and pulse height (right) distribution. The conditions: STM, 3,5x3,5 $\mathrm{mm}^{2}$; LYSO crystal, $3 \times 3 \times 15 \mathrm{~mm}^{3}$; VT120C amplifier, Na22 radioactive source. 334 ps (sigma) time resolution, $13.5 \%$ (FWHM) photo peak resolution.

\section{References}

1. A. Ronzhin, M. Albrow, K. Byrum, M. Demarteau, S. Los, E. May, E. Ramberg, J. Vav'ra, A. Zatserklyaniy. Tests of Timing Properties of Silicon Photomultipliers. Nucl. Instr. and Meth. A 616 (2010) 38.

2. A. Ronzhin, M. Albrow, K. M. Demarteau, S. Los, A.S. Pranko, S. Malik, E. Ramberg, A. Zatserklyaniy. Development of 10 Picosecond Level Time of Flight System in the Meson Test Beam at Fermilab. Nucl. Instr. and Meth. A 623 (2010) 931-941

3. M. Mazzillo, G. Condorelli, D. Sanfilippo, G. Valvo, B. Carbone, A. Piana, G. Fallica, A.
Ronzhin, M. Demarteau, S. Los, E. Ramberg. Timing Performances of Large Area Silicon Photomultipliers fabricated at STMicroelectronics. IEEE Trans. Nucl. Sci., vol. 57, no. 4, Aug. 2010, 2273-2279.

4. M. Mazzillo et al., "Silicon photomultiplier technology at STMicroelectronics", IEEE Trans. Nucl. Sci., vol. 56, no 4, pp. 24342442, Aug. 2009.

5. S. Ritt. Design and Performance of the $6 \mathrm{GHz}$ Waveform Digitizing Chip DRS4. http://drs.web.psi.ch/docs/nss08.pdf

6. C. L. Kim, G. C. Wang, S. Dolinsky. MultiPixel Photon Counters for TOF-PET Detectors and its challenges, IEEE Trans. Nucl. Sci., vol. 56, no. 5, October 2009.

7. P. Jarron, E. Auffray, S. E. Brunner, M. Despeisse, E. Garutti, M. Goettlich, H. Hillemanns, P. Lecoq, T. Meyer, F. Povolny, W. Shen, H. C. Schultz-Coulon, C. Williams. Time based readout of silicon photomultiplier (SiPM) for Time Of Flight Positron Emission Tomography (TOF-PET) http://infoscience.epfl.ch/record/143994/file s/preprint_536.pdf

8. D. R. Schaart, H. T. van Dam,S. Seifert, R. Vinke, P. Dendooven, H. Lohner, F. J. Beekman. A novel, SiPm-array-based, monolithic scintillator for PET, Phys. Med. Biol. 54, 2009, 3501-3512

9. D. R. Schaart, S. Seifert, R. Vinke, H. van Dam $^{1}$, P. Dendooven, H. Löhner F. Beekman' $\mathrm{LaBr}_{3}$ :Ce and SiPMs for time-offlight PET: achieving 100 ps coincidence resolving time, 2010 Phys. Med. Biol. 55 N179

10. http://www.keithley.com/products/dcac/volt agesource/highvoltage/?mn=2410

11. E. Ramberg, A. Ronzhin, M. Albrow, S. Los, A. Pranko, S. Malik, A. Zatserklyaniy. "Photodetector Timing Research at Fermilab". Acta Physica Polonica B, Proceeding Supplement, Vol. 4 (2011) 1066. 
